\title{
Model Checking without Hardware Drivers
}

\author{
Carlos M. Roman, Gary De Palma \\ Bell Labs Design Automation \\ Robert Kurshan \\ Bell Labs \\ Lucent Technologies, Inc. \\ 600-700 Mountain Ave, P.O. Box 636, \\ Murruy Hill, NJ USA 07974-0636 USA
}

\begin{abstract}
Regardless of whether a simulator or a model checker is used for functional verification, proper capture of the design environment is one of the most problematic steps. This step is limited not just to the proper definition of Clock and Reset pins, but includes the specification of all combinational and sequential input constraints required to establish a reasonable interface to verify the design. Underconstraining the inputs leads to spurious failures, requiring tedious iterations of the environment model. What is worse, if the environment overconstrains the inputs, then potentially fatal input sequences will be overlooked. Since verification addresses all possible behaviors, the issue of a correct and adequate environment model is more critical, and thus requires more precision than the conventional approach used for simulators. In this paper, we address these fundamental differences as they are applicable to FormalCheck[Tm], an $\omega$-automata based model checker comerciallized by Bell-Labs Design Automation, a divisions of Lucent Technologies Inc.

The level of expressiveness in FormalCheck's automata constructs allow for efficient capture of design environments. These constructs cover the entire spectrum from assumptions and exclusions to eventualities, making the generation of safety and fairness constraints straight-forward. More importantly, these automata constructs can eliminate the need for problematic drivers (input conditioners/controllers) and monitors (output loggers/observers) altogether.
\end{abstract}

